\title{
Local earth magnetic field and ischemic heart disease: peculiarities of interconnection
}

\author{
Greta Žiubrytè ${ }^{1}$, Vaiva Šiaučiūnaitè ${ }^{2 *}$, Gediminas Jaruševičius ${ }^{3}$ and Rollin McCraty ${ }^{4}$ \\ ${ }^{1}$ Faculty of Medicine, Academy of Medicine, Lithuanian University of Health Sciences, Kaunas, Lithuania \\ ${ }^{2}$ Department of Mathematical Modelling, Kaunas Technology University, Lithuania \\ ${ }^{3}$ Department of Cardiology, Hospital of Lithuanian University of Health Sciences Kaunas Clinics, Kaunas, Lithuania \\ ${ }^{4}$ HeartMath Institute, California, USA
}

\begin{abstract}
Ischemic heart disease (IHD), in clinical practice manifesting as acute coronary syndrome (ACS), might be understood as a disruption of intracoronary blood flow decreasing oxygenated blood supply to the heart muscle. Oscillations of the local time varying magnetic field (TVMF) effects such human cardiovascular system indicators as blood pressure (BP), heart rate (HR) and its variability. This might be the foundation for magnetic activity association to increased occurrence of IHD and myocardial infarction (MI). An attempt is being made to discover new promising methods to reduce and prevent cardiovascular disease and its complications. Methods: Seven-hundred patients admitted to Cardiology Department of Hospital of Lithuanian University of Health Sciences Kaunas Clinics within 2016 due to acute coronary syndrome were retrospectively included into the study. The number of cases per week was compared with the weekly changes of the local Earth magnetic field. Results: Significant correlations between weekly occurrences of ACS cases and the average weekly TVMF strength were found. In the analyses of the whole year model two positive weak and moderate correlation coefficients in SBeta [15;32] $(r=0.06, p=0.65)$ and SGamma [32;65] ( $r=0.27$, $p=0.05)$ ranges in the female group were found, respectively. In males there was no significant correlations in analyses of the whole year model. When one-year period was divided into twotime intervals, further analyses revealed that the first-half of the year had the same tendencies in the females with a single positive moderate correlation coefficient in SGamma [32;65] range ( $\mathrm{r}=0.28, \mathrm{p}=0.05)$ (Figure 2 A.). In males there were tendencies towards weak to moderate positive correlation coefficients ( $\mathrm{p}<0.1)$ at all frequency ranges in the second half of the year, which are detailed in Figure 2 B. There were no differences between ACS cases in male and female though the secondhalf of the year. Conclusions: Significant correlation between acute coronary syndrome and the local Earth magnetic field changes was revealed. The acute coronary syndrome is positively correlated with the local Earth magnetic field in SGamma range in female through the year. A higher magnetic field in SBeta and SGamma ranges is associated with higher incidences of acute coronary syndrome through the year in females. The higher magnetic field in SGamma range is associated with higher incidences of acute coronary syndrome through the year in females and through the second-half of the year in males.
\end{abstract}

\section{Introduction}

Ischemic heart disease (IHD), in clinical practice manifesting as acute coronary syndrome (ACS), might be understood as a disruption of intracoronary blood flow decreasing oxygenated blood supply to the heart muscle. In pathogenesis of IHD the coronary artery narrowing due to atherosclerotic plaques formation and clotting disorders are fundamental. It is a relevant problem in Lithuania as in addition to decease of more than half citizens' annually, it affects even younger patient in recent years. It is suggested that geomagnetic activity in addition to human humoral activity regulation [1] is significantly associated with mortality from cardiac diseases and strokes. Cosmic radiation and Solar activity's correlations with suicides, transport injuries and onsets of non-cardiac diseases have been demonstrated. [2]. Oscillations of the local time varying magnetic field (TVMF) effects such human cardiovascular system indicators as blood pressure (BP) $[3,4]$, heart rate (HR) and its variability. [5]. This might be the foundation for magnetic activity association to increased occurrence of IHD and myocardial infarction (MI). An attempt is being made to discover new promising methods to reduce and prevent cardiovascular disease and its complications.

\section{Methods}

Study population: In total $700(425(60,7 \%)$ male and $275(39,3 \%)$ female) patients admitted to the Cardiology Department of Hospital of
Lithuanian University of Health Sciences Kaunas Clinics due to ACS between 1 January 2016 and 31 December 2016 were included into the study. The mean age of male was $65,5( \pm 11,3)$ years and $73,1( \pm 9,9)$ years for female.

Magnetometer Data: The TVMF intensity was measured by the Global Coherence Monitoring Network magnetometer located In Lithuania. Hourly data was downloaded to a personal computer and transformed into successive thirty-second length segments for each of whom the power spectral density (PDS) was calculated. These hourly PDS segments were averaged together and the sum of them in $0-65 \mathrm{~Hz}$ frequency range was calculated within the study period. The local Earth's magnetic field was observed in five frequency intervals overlapping with EEG $[\mathrm{Hz}]$, but to stress that those frequency resonances are obtained from Schumann resonances we add S in front: SDelta [0;3,5], STheta [3,5;7], SAlpha [7;15], SBeta [15;32], SGamma [32;65]. We added [0;65] where all frequency intervals were summarised in one.

${ }^{*}$ Correspondence to: Vaiva Šiaučiūnaitė, Department of Mathematical Modelling, Kaunas Technology University, Lithuania, E-mail: vaiva91@gmail.com

Key words: ischemic heart disease, acute coronary syndrome, the local Earth magnetic field

Received: June 15, 2018; Accepted: June 22, 2018; Published: June 27, 2018 
Statistical Analysis: The statistical analysis was performed using the software package SPSS 20.0. Nonparametric Mann-Whitney $\mathrm{U}$ test was used for the comparison of two independent samples. Pearson correlation coefficient for the linear correlation between two variables was calculated. The level of $p<0.05$ was considered statistically significant.

\section{Results}

Significant correlations between weekly occurrences of ACS cases and the average weekly TVMF strength were found. In the analyses of the whole year model two positive weak and moderate correlation coefficients in SBeta [15;32] $(\mathrm{r}=0.06, \mathrm{p}=0.65)$ and SGamma [32;65] $(\mathrm{r}=0.27, \mathrm{p}=0.05)$ ranges in the female group were found, respectively. In males there was no significant correlations in analyses of the whole year model. These correlations are presented in Figure 1.

When one-year period was divided into two-time intervals, further analyses revealed that the first-half of the year had the same tendencies in the females with a single positive moderate correlation coefficient in SGamma [32;65] range $(\mathrm{r}=0.28, \mathrm{p}=0.05)$ (Figure 2A). In males there were tendencies towards weak to moderate positive correlation coefficients $(p<0.1)$ at all frequency ranges in the second half of the year, which are detailed in Figure 2B. There were no differences between ACS cases in male and female though the second-half of the year.

\section{Conclusion}

1. Significant correlation between acute coronary syndrome and the local Earth magnetic field changes was revealed.

2. The acute coronary syndrome is positively correlated with the local Earth magnetic field in SGamma range in female through the year.

3. A higher magnetic field in SBeta and SGamma ranges is associated with higher incidences of acute coronary syndrome through the year in females.

4. The higher magnetic field in SGamma range is associated with higher incidences of acute coronary syndrome through the year in females and through the second-half of the year in males.

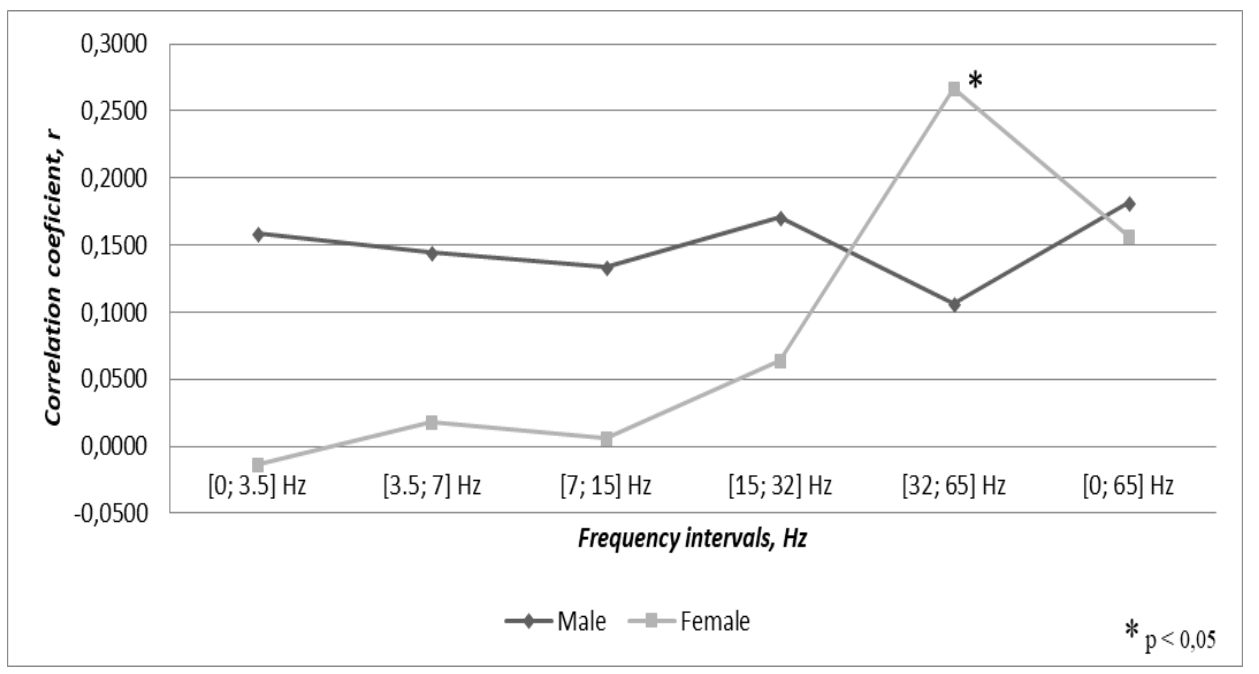

Figure 1. Correlation between gender related cases of acute coronary syndrome and the time varying magnetic field changes through the year
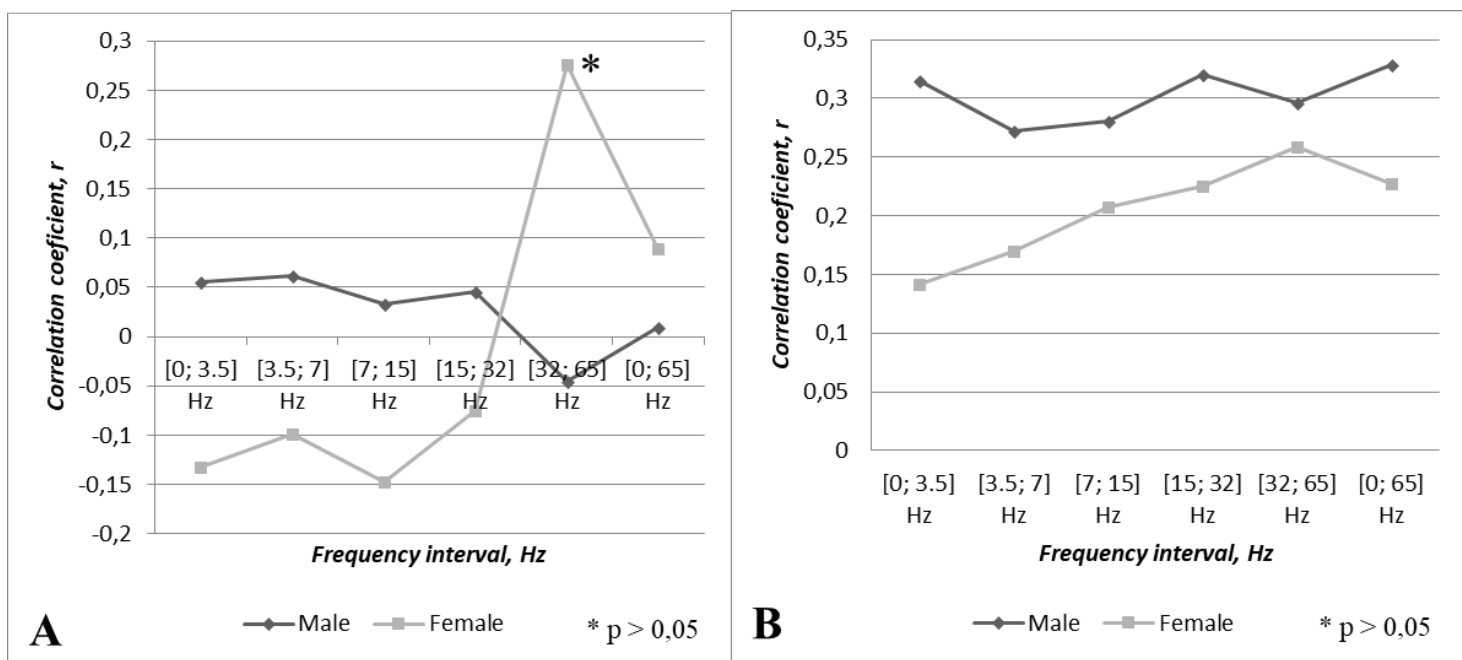

Figure 2. Correlation between gender related cases of acute coronary syndrome and the time varying magnetic field changes through the first-half of the year (A) and through the secondhalf of the year (B) 


\section{References}

1. Hedayat K. Systems Biology Reaserch Group (2017). Available at: https://www. facebook.com/plugins/post.php?href=https $\% 3 \mathrm{~A} \% 2 \mathrm{~F} \% 2 \mathrm{Fwww}$.facebook.com $\% 2 \mathrm{Fsyst}$ emsbiologyresearchgroup \%2Fposts\%2F300204793813215\&width $=500$

2. Stoupel E, Israelevich P, Petrauskiene J, Kalediene R, Abramson E, et al. (2002) Cosmic rays activity and monthly number of deaths: a correlative study. $J$ Basic Clin Physiol Pharmacol 13: 23-32. [Crossref]
3. Stoupel E, FESC (2006) Cardiac Arrhythmia and Geomagnetic Activity. Indian Pacing Electrophysiol J 6: 49-53 [Crossref]

4. Stoupel E (2002) The effect of geomagnetic activity on cardiovascular parameters. Biomed Pharmacother 56: 247-256. [Crossref]

5. Cornélissen G (2002) Non-photic solar associations of heart rate variability and myocardial infarction. Journal of Atmospheric and Solar-Terrestrial Physics 707720

Copyright: (C2018 Žiubrytė G. This is an open-access article distributed under the terms of the Creative Commons Attribution License, which permits unrestricted use, distribution, and reproduction in any medium, provided the original author and source are credited. 